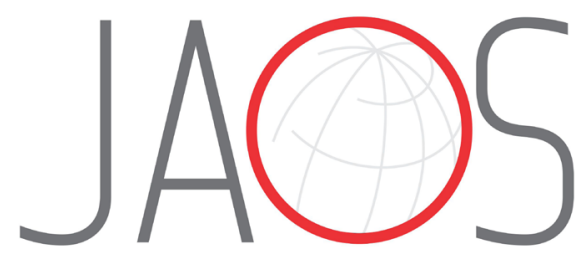

JOURNAL OF APPLIED ORAL SCIENCE

\title{
The prevalence of novel periodontal pathogens and bacterial complexes in Stage II generalized periodontitis based on 16S rRNA next generation sequencing
}

Abstract

Salem ABU FANAS ${ }^{1}$

Carel BRIGI ${ }^{2}$

Sudhir Rama VARMA ${ }^{1}$

Vijay DESAI ${ }^{1}$

Abiola SENOK ${ }^{3}$ Jovita D'SOUZA ${ }^{1,4}$ iD
Submitted: September 15, 2020 Modification: January 9, 2021

Accepted: February 10, 2021
Objective: To define the subgingival microbial profile associated with Stage II generalized periodontitis using next-generation sequencing and to determine the relative abundance of novel periodontal pathogens and bacterial complexes. Methodology: Subgingival biofilm samples were collected from 80 subjects diagnosed with Stage II generalized periodontitis. Bacterial DNA was extracted, and 16S rRNA-based bacterial profiling via next-generation sequencing was carried out. The bacterial composition and diversity of microbial communities based on the age and sex of the patients were analyzed. The bacterial species were organized into groups: bacterial complexes (red, orange, purple, yellow, and green), novel periodontal pathogens, periodontal health-related species, and unclassified periodontal species. The results were analyzed and statistically evaluated. Results: The highest number of bacteria belonged to the phylum Bacteroidetes and Firmicutes. In terms of relative abundance, the orange complex represented $18.99 \%$, novel bacterial species (Fretibacterium spp. and Saccharibacteria spp.) comprised $17.34 \%$, periodontal health-related species accounted for $16.75 \%$ and unclassified periodontal species represented (Leptotrichia spp. and Selenomonas spp.) $15.61 \%$. Novel periodontal pathogens had outweighed the periodontal disease-related red complex (5.3\%). The one-sample z-test performed was statistically significant at $p<0.05$. The Beta diversity based on the unweighted UniFrac distance at the species level demonstrated a total variance of $15.77 \%$ based on age and $39.19 \%$ on sex, which was not statistically significant. Conclusion: The bacterial species corresponding to the disease-related orange complex and novel periodontal pathogens are predominant in Stage II generalized periodontitis.

Keywords: Periodontal pathogen. 16S rRNA. Next-Generation sequencing. Microbial profile.
Center of Medical and Bio-allied Health Sciences research, Ajman University, Ajman, UAE.

${ }^{2}$ Ajman University, College of Dentistry, Department of General Dentistry, Ajman, United Arab Emirates.

${ }^{3}$ Mohammed Bin Rashid University of Medicine and Health Sciences, College of Medicine, Department of Microbiology.

${ }^{4}$ Gulf Medical University, Department of Periodontics, College of Dentistry, Ajman, UAE. 


\section{Introduction}

Periodontitis is a biofilm-associated inflammatory disease of the periodontium characterized by the destruction of periodontal tissue. Three significant factors are involved in the pathogenesis of periodontitis: susceptible host, presence of periodontal pathogens, and reduction or absence of beneficial bacterial species for periodontal health. Whilst, the microbial composition of subgingival biofilm differs in healthy individuals, the development of periodontitis is directly co-related to a characteristic shift in the microbiome referred to as "Dysbiosis." ${ }^{1,2}$ The inter-relationship between dysbiosis of the oral microbiome and the periodontitis progression has been established by several studies. ${ }^{3,4}$ These investigations postulate the role of the microbiome on the onset and progression of periodontitis.

The role of microbiome relating to periodontal health and disease was first described by Socransky, et al. ${ }^{5}$ (1998). This path-breaking study - using whole genomic DNA probes to 40 bacterial species - has defined the potential role of the bacterial complex as opposed to individual bacterial species. The red complex is composed of three bacterial species: Treponema denticola, Porphyromonas gingivalis, and Tannerella forsythia that were associated with periodontal disease. The yellow complex composed of different Streptococcus species and green complex composed of Capnocytophaga species were firmly related to periodontal health. The orange complex - including Fusobacteria species - members of Prevotella, and Campylobacter were classified as periodontopathogens. The detection and quantitation of these bacterial complexes were described with whole genomic DNA probes, checkerboard DNA-DNA hybridization, ${ }^{5}$ and using highly sensitive techniques such as real-time PCR (polymerase chain reaction). ${ }^{6}$ These test may also be used to quantify species from Socransky complexes.

However, recent investigations using metagenomics and metatranscriptomics proposed that more diverse periodontitis-associated microbiota are involved in periodontal disease. According to this model, periodontitis results from polymicrobial synergy and dysbiosis that disturbs the ecologically balanced biofilm associated with periodontal tissue homeostasis. ${ }^{7}$ In this model, the host's immune response is initially disrupted by keystone pathogens supported by accessory pathogens and it is subsequently overactivated by pathobionts resulting in homeostasis breakdown and destructive inflammation. The host's immune response is dysregulated either because it is disrupted by the microbial community or due to host immunoregulatory defect, resulting in bacterial outgrowth and overt pathogenicity. ${ }^{8}$

Recent studies have also identified novel periodontal pathogens and additional bacterial species associated with periodontal health and disease. A study conducted by Kirst, et al. ${ }^{9}$ (2015) has confirmed the presence of Rothia and Streptococcus species related to periodontal health. Similarly, investigations based on next-generation sequencing has revealed the presence of novel periodontal pathogens/pathobionts like ${ }^{10,11,12}$ Fretibacteirum fastidiosum, Filifactor alocis, and Eubacterium saphenum. A recent review of the role of bacterial biofilm in periodontal diseases has highlighted the presence of novel pathogens and health-related species in the subgingival biofilm. The review based on next generation sequencing studies has categorized Fretibacterium spp., Saccharibacteria spp., Dialister spp., as novel pathogens and Rothia spp., Kingella spp., Gemella spp., Corynebacterium spp. as health-related species. ${ }^{13}$

Despite some studies revealing the presence of novel bacterial species, evidence reporting their relative prevalence regarding established bacterial complexes are rare. $16 \mathrm{~S}$ rRNA-based bacterial method constitute an efficient method to determine the composition and diversity of bacterial communities. ${ }^{14}$ Therefore, this study aims to illustrate the subgingival bacterial profile associated with Stage II generalized periodontitis using NGS of 16S RNA gene, and to identify the relative abundance of novel periodontal pathogens and bacterial complexes.

\section{Methodology}

\section{Study population}

This investigation was developed in compliance with the Helsinki Declaration on Medical Research Involving the Ethics Committee at Ajman University (F-H17-11-03). The study was registered under clinical trial with the identification number as NCT04425343. Prior to the participation in the study, informed consent from all the participants was obtained. The study was conducted from September 2018 to November 
2019. The included participants had no history of systemic diseases (hypertension, diabetes mellitus, etc.) and they had not received any antibiotics three months prior to sampling. Exclusion criteria included pregnancy, history of smoking, and periodontal therapy over the past three months. Patients recruited for the study were diagnosed as Stage II Generalized periodontitis according to the classification defined at the 2017 world workshop: "classification of periodontal and peri-implant disease and conditions." ${ }^{15}$ Periodontal status presenting a severity of interdental clinical attachment loss of 3-4 mm, radiographic bone loss between $15 \%-33 \%$, and subjects with absence of tooth loss were included. Patients with periodontal pocket of $\leq 5 \mathrm{~mm}$ with horizontal bone loss and having an extent and distribution with $>30 \%$ teeth involved were selected. A total of 80 subgingival biofilm samples were collected from 36 females and 44 males, aged from 25 to 39 years. Among the total participants, 28 patients (12 females; 16 males) were in the age group of $25-32 y r s$ and 52 patients ( 24 females; 28 males) aged from 33 to $39 y$ rs. The buccal aspect of maxillary molars and mandibular molars were isolated with cotton rolls and air dried gently to discard the supragingival plaque with curettes. Using a sterile Gracey curette no 9-10, 11-12 (Hu-Friedy, USA), subgingival biofilm was removed from the base of periodontal pocket with slight pressure and collected to the level of $0.1 \mathrm{~mL}$ in $1.5 \mathrm{~mL}$ microcentrifuge tube containing $200 \mu \mathrm{L}$ of sterile Buffer CL (ComplexioLyte buffer - for solubilization of membrane proteins and protein complexes in bacteria).

\section{DNA extraction, library construction, sequencing, and data processing}

DNA extraction was carried out using the QIAmp ${ }^{\circledR}$ DNA (Qiagen, Hilden, Germany) extraction kit according to the manufacturer's guidelines. After measuring DNA purity, the amount of DNA per sample was quantified using the DeNovix dsDNA Broad Range Assay kit (DeNovix, Wilmington DE, USA), and samples had a concentration of $36 \pm 14 \mathrm{ng} / \mu \mathrm{L}$ for downstream workflow. For next generation sequencing, we amplified the V3-V4 region of the 16S rRNA gene using the Taq PCR Master Mix Kit (Qiagen). The full-length primer sequences, using standard IUPAC nucleotide nomenclature, to follow the protocol targeting this region were:

16SAmpliconPCRForwardPrimer $=5^{\prime}$

TCGTCGGCAGCGTCAGATGTGTATAAGAGA

\section{CAGCCTACGGGNGGCWGCAG}

16SAmpliconPCRReversePrimer $=5^{\prime}$

GTCTCGTGGGCTCGGAGATGTGTATAAGAGA CAGGACTACHVGGGTATCTAATCC

Aliquots of the PCR product were assessed on the Fragment Analyzer (Advanced Analytical, Parkersburg WV, USA) and purified using Agencourt AMPure XP beads (Beckman Coulter, Atlanta GA, USA) and quantified using the DeNovix dsDNA High Sensitivity Assay kit (DeNovix) following manufacturer's protocols. Unique oligonucleotide indexes were added to the amplicons using the Nextera XT Index Kit (Illumina, San Diego, USA), indexed amplicons were purified using Agencourt AMPure XP beads (Beckman Coulter) and fragment lengths were assessed on the Fragment Analyzer using High Sensitivity Small Fragment Analysis Kit (Advanced Analytical). Up to 96 sequencing libraries with unique Nextera XT Indexes were pooled in equimolar concentrations in a single reaction tube and sequenced on the MiSeq instrument (Illumina) using the MiSeq Reagent 600 cycle v3 kit (Illumina). The bioinformatics pipeline used for processing microbiome $16 \mathrm{~S}$ sequence data was QIIME (Quantitative Insights into Microbial Ecology). The bioinformatics pipeline consisted of five main steps, as described in Figure 1 . Sequences shorter than 200 bp, indicating a Phred score lower than 20 were removed using the QIIME2 dada2 denoisepaired module. The remaining sequence data was used for de novo binning of operational taxonomic units (OTUs) based on sequence similarity followed by selection of representative OTUs per bin. All the clean tags from 80 samples were clustered into OTUs using QIIME at $97 \%$ sequence similarity. The $16 \mathrm{~S}$ Metagenomics workflow classified organisms based on V3 and V4 amplicon utilizing a database of $16 \mathrm{~S}$ rRNA data. Classification of reads at several taxonomic levels was based on Greengenes database (http:// greengenes.lbl.gov/). Multiple sequence alignment of representative sequences and microbial community profiling was done using QIIME2. Estimation of alpha and beta diversity was carried out based on the operational taxonomic units.

Bacterial species were sorted into groups in order to obtain the relative abundance of bacterial complexes and novel periodontal pathogens. The data collected with QIIME analysis at level seven was classified into their group, and the bacterial species that had no established role in periodontitis and prevalence 


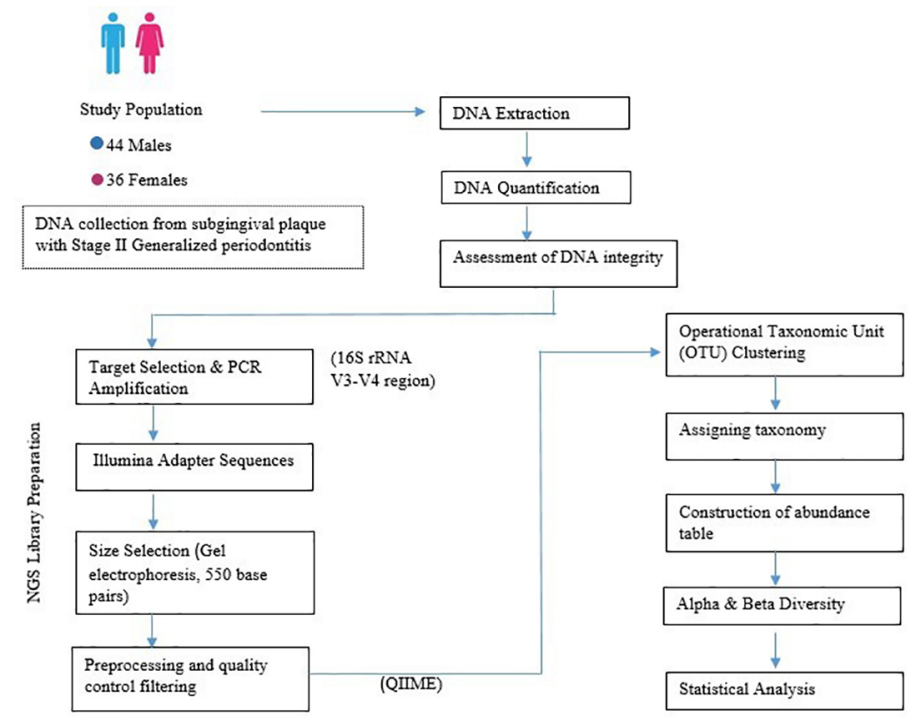

Figure 1- Experimental design and workflow for microbial profiling in samples diagnosed as Stage II generalized periodontitis

inferior to $0.01 \%$ were excluded. The species were classified based on their bacterial complexes (red, orange, purple, yellow, and green), novel periodontal pathogens, health-related species, and health/disease species. ${ }^{5,13}$ Health/disease species group were neither categorized as health nor disease species due to the lack of evidence supporting their role as periodontal pathogens/health species. ${ }^{13,16}$ The percentage of total abundance in each group was estimated by combining the abundance of individual species in each group. The relative abundance of each group for all subgingival biofilm samples was graphically plotted.

\section{Statistical analysis}

The data were entered using Microsoft Excel 2016 and imported into IBM SPSS Statistics 26 (IBM Corporation, New York, NY, United States) for statistical analysis. For investigating the means for different phylum and bacterial complexes a onesample t-test was performed at $p<0.05$. Furthermore, all quantitative variables related to clinical parameters were determined using Levene's Test for equality of variances. The evaluation of microbial community among all the biofilm samples for similarity was determined using Principal coordinates analysis (PCoA) performed at the species level. The intraand inter-group similarity based on the Unweighted UniFrac distance - which represents the phylogenetic measurement of beta diversity - was estimated using QIIME. The Spearman's correlation coefficient was used to analyze the difference between the Unweighted UniFrac distances among two groups (based on the age and sex) and significance was set at $\mathrm{p}<0.05$.

\section{Results}

\section{Sequencing}

The Illumina MiSeq sequencing reinstated a total of $9,316,880$ filtered clean tags, with an average of $114,461 \pm 2,570$ sequences per sample. The OTU richness varied from 271 to 580 per sample. Figure 2 presents rarefaction curves showing the observed OTU richness ( $97 \%$ sequence similarity as cutoff) with increasing sequence depth. All subgingival biofilm samples have attained a plateau, as depicted in the graph by the rarefaction curves obtained from OTUs. The asymptotic curves expressed in Figure 2 revealed that the sampling quality was excellent, indicating the probability that the addition of new species to the final samples has reached zero.

\section{Microbial taxonomic composition}

Figure 3 depicts nine phyla, which were commonly present in all samples diagnosed as Stage II generalized periodontitis. The phylum of Bacteroidetes exhibited a high prevalence, followed by Firmicutes representing $34.15 \%$ and $25.14 \%$, respectively. A statistically significant result in the mean scores for Bacteroidetes $(M=34.15 \pm 5.3)$ and for Firmicutes $(M=25.14 \pm 3.8)$ was observed at conditions $t(79)$ $=57.055 \mathrm{p}<0.05, \mathrm{t}(79)=59.14, \mathrm{p}<0.05$ respectively. The phylum including Spirochetes $8.60 \%$, Fusobacteria $7.50 \%$, Proteobacteria 7.33\%, and Actinobacteria $6.18 \%$ represented a similar frequency. Statistically significant results were also obtained for Spirochetes $(M=8.60 \pm 4.3)$ at $\mathrm{t}(79)=17.681 ;$ Fusobacteria $(M=7.50 \pm 2.55)$ at $t(79)=26.24$; Proteobacteria 
$(M=7.33 \pm 5.1)$ at $t(79)=13.01$ and Actinobacteria $(M=6.18 \pm 3.8)$ at $t(79)=12.50$ for $p<0.05$. The remaining phyla consisting of Patescibacteria 3.95\%, Epsilonbacteraeota 2.65\%, and Synergistetes 1.7\% constituted the minority with their statistical significant figures being $(M=3.95 \pm 1.8)$ at $t(79)=19.26$; $(M=2.65 \pm 1.2)$ at $t(79)=18.75 ;(M=1.95 \pm 0.8)$ at $t$ $(79)=20.47$ at $p>0.05$ respectively. The phyla group mentioned as "others" consisted of Chloroflexi,
Elusimicrobia, and Verrucomicrobia. The species of bacteria in these groups of phyla were not considered in this study as they were not consistently present in all samples, also, they represented a frequency inferior to $1 \%$. Levene's test for equality of variances for our analysis was $F(2,78)=1.49, p=0.226$. Since the significance was greater than 0.05, the Levene's Test was non-significant, and homogeneity of variance at phylum level were observed for age and sex.

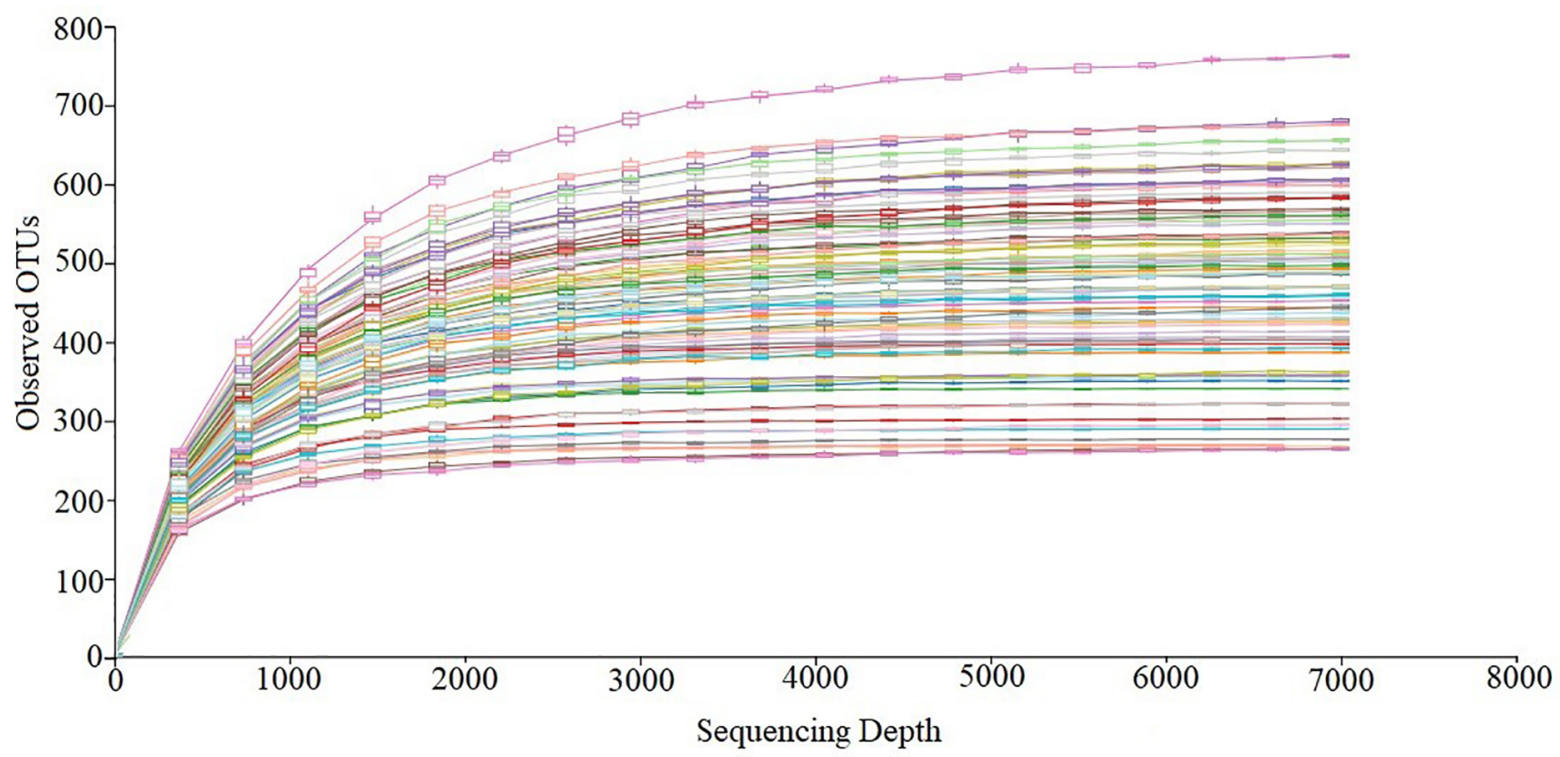

Figure 2- Represents rarefaction curves for the number of operational taxonomic units in each sample. Each sample received a separate color code representing its corresponding number of OTUs. The rarefaction curve based on $97 \%$ similarity were each color code given to 80 samples

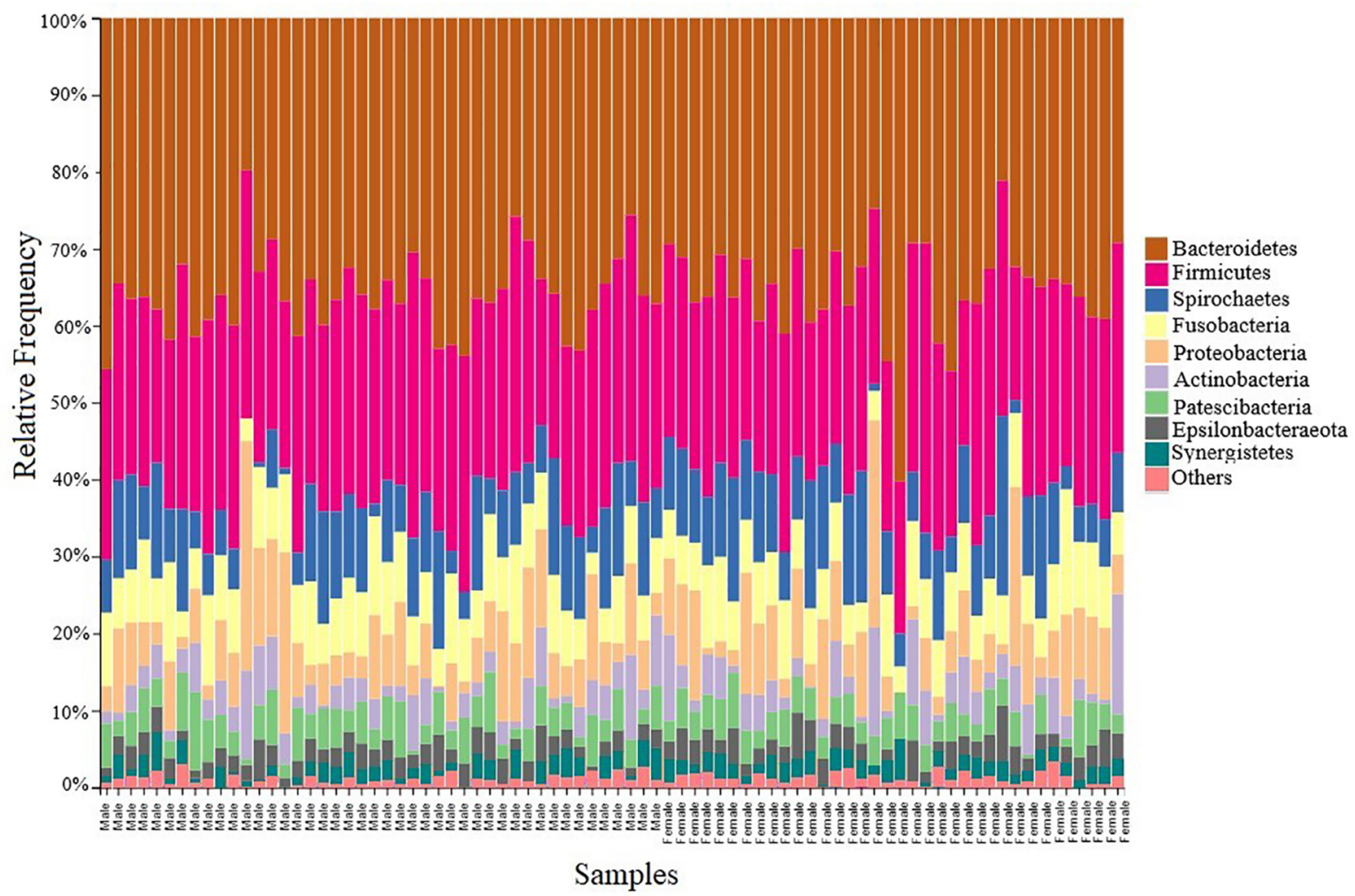

Figure 3- The relative frequency of the taxonomic composition in all the 80 sub-gingival biofilm samples. The bars are organized according to patients' sex. The group mentioned as others refer to the phylum presenting values below $1 \%$ 
Prevalence of bacterial class in stage II generalized periodontitis

The relative abundance of the different classes of bacteria were analyzed by computing scatter plots. The $\mathrm{X}$-axis in the graph representes the total count of bacteria in each class and $\mathrm{Y}$-axis their corresponding prevalence as fraction per samples. The correlation between presence of these bacterial classes and their incidence in Stage II periodontitis, was analyzed. All 15 bacterial classes presented tight clusters in an upward pattern curve (Figure 4). The tighter clustering represented a stronger relationship between the presence of each bacterial class in all samples diagnosed as Stage II periodontitis. Outliers were seen in case of Bacilli, Clostridia, and Bacteroidia but were relatively insignificant. The most prevalent class of bacteria per sample were Bacteroidia, Negativicutes, Spirochaetes, and Fusobacteria. The least predominant were Gracilibacteria, Coriobacteria, and Alphaproteobacteria bacterial class representing a frequency of less than 0.25 fraction per sample. The phyla, class, genus, and species isolated from the subgingival biofilm of all 80 subjects diagnosed as Stage II generalized periodontitis are shown in Figure 5.

\section{Abudance of microbial complexes and novel bacterial species}

Table 1 reveals the data obtained for the relative abundance of bacterial species sorted into nine groups. The one-sample z-test performed for each group was statistically significant at $p<0.05$. The $t$ statistics results are described in Table 1 . The periodontal disease species included for novel periodontal pathogens were Fretibacterium spp. and Saccharibacteria spp. The health-related species was composed of Neisseria spp., Gemella spp., Rothia spp., Kingella spp., and Corynebacterium spp. The group composed of Leptotrichia and Selenomonas species represented $15.61 \%$. The red complex composed of T. denticola, T. forsthyia and $P$. gingivalis represented a relative frequency of $5.3 \%$. The orange complex, novel-periodontal pathogen, and periodontal healthspecies indicated a significant value of $18.99 \%$, $17.34 \%$, and $15.61 \%$, respectively. Among the healthrelated complexes, the green complex $(1.33 \%)$ was

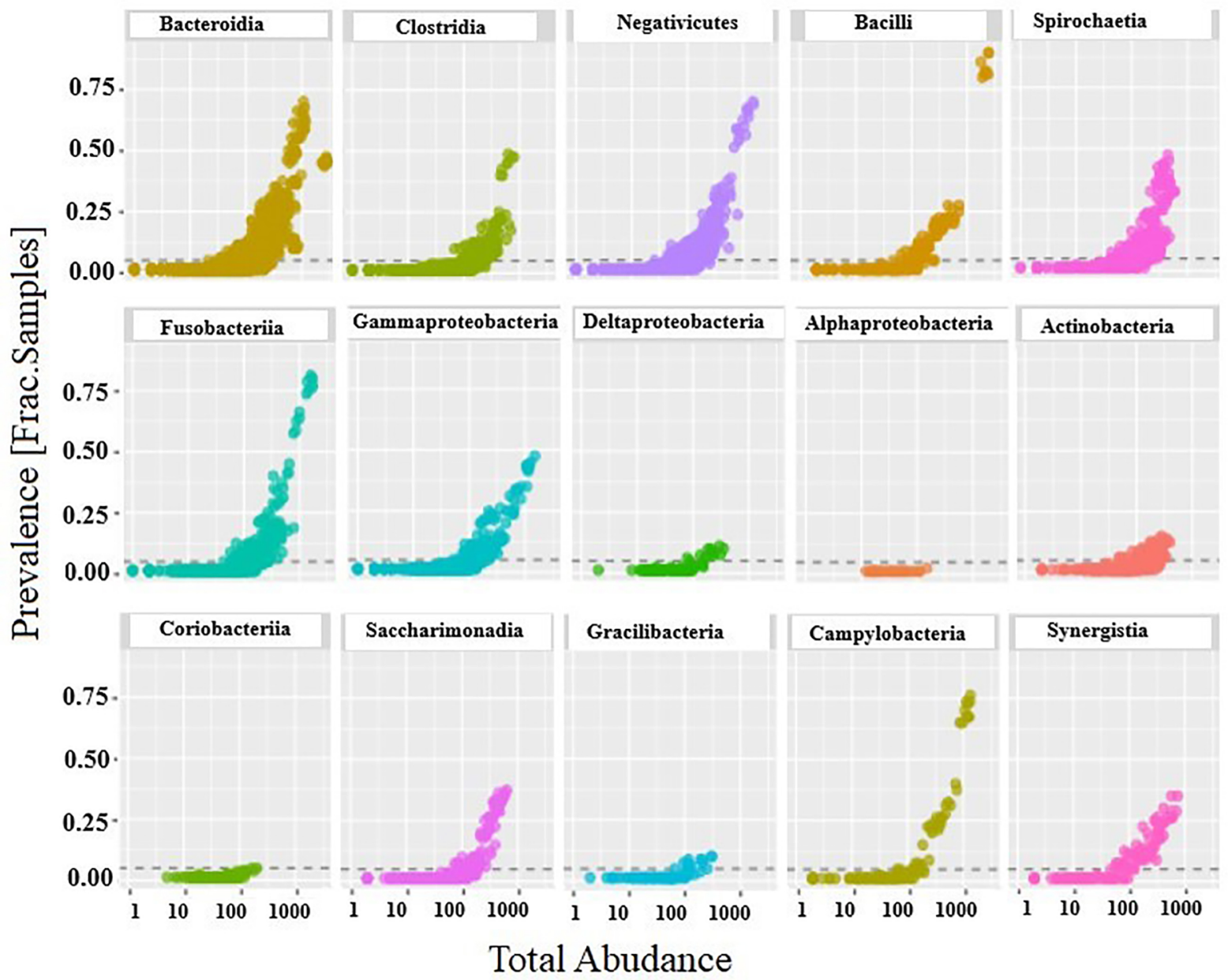

Figure 4- The prevalence of each bacterial class as fraction per sample plotted against their total abundance 


\begin{tabular}{|c|c|c|c|c|}
\hline No. & Phylum & Class & Genus & Species \\
\hline \multirow{27}{*}{1} & \multirow{27}{*}{ Bacteroidetes } & \multirow{27}{*}{ Bacteroidia } & \multirow{4}{*}{ Porphyromonas } & Porphyromonas endodontalis \\
\hline & & & & Porphyromonas gingivalis \\
\hline & & & & Porphyromonas sp. oral clone HF001 \\
\hline & & & & Porphyromonas catoniae \\
\hline & & & \multirow{13}{*}{ Prevotella } & Prevotella intermedia \\
\hline & & & & Prevotella oris \\
\hline & & & & Prevotella denticola \\
\hline & & & & Prevotella nigrescens \\
\hline & & & & Prevotella salivae \\
\hline & & & & Prevotella buccae \\
\hline & & & & Prevotella dentalis \\
\hline & & & & Prevotella veroralis \\
\hline & & & & Prevotella baroniae \\
\hline & & & & Prevotella sp. Oral Taxon 300 \\
\hline & & & & Prevotella maculosa \\
\hline & & & & Prevotella heparinolytica \\
\hline & & & & Prevotella zoogleoformans \\
\hline & & & \multirow{3}{*}{ Tannerella } & Tanneralla Forysthia \\
\hline & & & & Tannerella sp. HMT808 \\
\hline & & & & Tannerella sp. \\
\hline & & & \multirow{4}{*}{ Capnocytophaga } & Capnocytophaga Granulosa \\
\hline & & & & Capnocytophaga Haemolytica \\
\hline & & & & Capnocytophaga Ochracae \\
\hline & & & & Capnocytophaga Sputigena \\
\hline & & & \multirow{3}{*}{ Odoribacter } & Bacteroidia bacterium canine oral taxon 187 \\
\hline & & & & Bacteroidetes sp. oral clone FX069 \\
\hline & & & & Odoribacter denticanis \\
\hline \multirow{18}{*}{2} & \multirow{18}{*}{ Firmicutes } & \multirow{18}{*}{$\begin{array}{l}\text { Clostridia } \\
\text { Negativicutes } \\
\text { Bacilli }\end{array}$} & \multirow{2}{*}{ Gemella } & Gemella Haemolysans \\
\hline & & & & Gemellla Morbillorum \\
\hline & & & \multirow{6}{*}{ Streptococcus } & Streptococcus Angiosus \\
\hline & & & & Streptococcus Gordonii \\
\hline & & & & Streptococcus Massiliensis \\
\hline & & & & Streptococcus Intermedius \\
\hline & & & & Streptococcus Mitis \\
\hline & & & & Streptococcus Sanguis \\
\hline & & & \multirow{3}{*}{ Peptostreptococcos } & Peptostreptococcos micros \\
\hline & & & & Peptostreptococcos oral clone CK035 \\
\hline & & & & Peptostreptococcos sp. oral clone BSO44 \\
\hline & & & Johnsonella & Johnsella sp.HMTI66 \\
\hline & & & Oribacterium & Oribacterium $s p$. \\
\hline & & & Voillonollorea & Veillonella parvula \\
\hline & & & vemornemoced & Veillonella sp. oral clone X042 \\
\hline & & & & Selenomonas noxia \\
\hline & & & Selenomonas & Selenomonas Sputigena \\
\hline & & & & Selenomonas oral clone EW076 \\
\hline \multirow{11}{*}{3} & \multirow{11}{*}{ Spirochaetes } & & & Treponema denticola \\
\hline & & & & Treponema lecithinolyticum \\
\hline & & & & Treponema medium \\
\hline & & & & Treponema parvum \\
\hline & & & & Treponema pectinovorum \\
\hline & & Spirochaetes & Treponema & Treponema socranskii \\
\hline & & & & Treponema sp. l:Q:AT20 \\
\hline & & & & Treponema sp. canine oral taxon 087 \\
\hline & & & & Treponema sp. oral taxon 265 \\
\hline & & & & Treponema maltophilum \\
\hline & & & & Treponema vincentii \\
\hline & & & Fusohasterium & Fusobacterium nucleatum \\
\hline 4 & Fusobacteria & Fusobacteriia & rusonactermum & Fusobacterium fastidosum \\
\hline 4 & Toundactia & 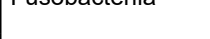 & I entotrichia & Leptotrichia wadei \\
\hline & & & Leptotrıcnia & Leptotrichia buccalis \\
\hline
\end{tabular}

Continued on the next page

Figure 5- Bacterial species present in all the biofilm samples and their corresponding genus, class, and phylum 
Continued from previous page

\begin{tabular}{|c|c|c|c|c|}
\hline \multirow{7}{*}{5} & \multirow{7}{*}{ Proteobacteria } & \multirow{7}{*}{$\begin{array}{l}\text { Gammaproteobcteria } \\
\text { Deltaproteobacteria } \\
\text { Alphaproteobacteria }\end{array}$} & Desulfobulbus & Desulfobulbus orale \\
\hline & & & \multirow{4}{*}{ Neisseria } & Neisseria sicca \\
\hline & & & & Neisseria mucosa \\
\hline & & & & Neisseria elongata \\
\hline & & & & Eikenella corrodens \\
\hline & & & \multirow{2}{*}{ Kingella } & Kingella Oralis \\
\hline & & & & Kingella Denitrificans \\
\hline \multirow{13}{*}{6} & \multirow{13}{*}{ Actinobacteria } & \multirow{13}{*}{ Actinobacteria } & \multirow{9}{*}{ Actinomyces } & Actinomyces cardiffensis F0333 \\
\hline & & & & Actinomyces gerencseriae DSM 6844 \\
\hline & & & & Actinomyces graevenitzii F0530 \\
\hline & & & & Actinomyces israelii \\
\hline & & & & Actinomyces massiliensis 4401292 \\
\hline & & & & Actinomyces meyeri \\
\hline & & & & Actinomyces odontolyticus \\
\hline & & & & Actinomyces sp. oral taxon 414 \\
\hline & & & & Actinomyces sp. oral taxon 448 str. F0400 \\
\hline & & & Bifidobacterium & Bifidobacterium longum subsp. Longum \\
\hline & & & \multirow{2}{*}{ Corynebacterium } & Corynebacterium durum \\
\hline & & & & Corynebacterium matruchotii \\
\hline & & & Scardovia & Scardovia wiggsiae F0424 \\
\hline \multirow{5}{*}{7} & \multirow{5}{*}{ Patescibacteria } & \multirow{5}{*}{$\begin{array}{l}\text { Saccharimonadia } \\
\text { Gracilibacteria }\end{array}$} & \multirow{2}{*}{ Gracilibacteria } & Gracilibacteria bacterium oral taxon 873 \\
\hline & & & & Gracilibacteria bacterium oral taxon 871 \\
\hline & & & Saccharibacteria & Candidatus Saccharibacteria bacterium UB2523 \\
\hline & & & 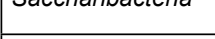 & Saccharibacteria spp. \\
\hline & & & Saccharimonades & Uncultured bacterium \\
\hline \multirow{3}{*}{8} & \multirow{3}{*}{ Epsilonbacteraeota } & \multirow{3}{*}{ Campylobacteria } & \multirow{2}{*}{ Campylobacter } & Campylobacter rectus \\
\hline & & & & Campylobacter gracilis \\
\hline & & & Wolinella & Wolinella rectus \\
\hline \multirow{3}{*}{9} & \multirow{3}{*}{ Synergistetes } & \multirow{3}{*}{ Synergistia } & \multirow{3}{*}{ Fretibacterium } & Fretibacterium fastidiosum \\
\hline & & & & Fretibacterium sp. \\
\hline & & & & Fretibacterium sp. \\
\hline
\end{tabular}

minimally present compared to the yellow (7.49\%) and purple $(7.9 \%)$ complexes. The relative abundance of Socransky's bacterial complexes and novel bacterial species in all samples are depicted in Figure 6.

\section{Alpha and beta diversity}

Chao $^{1}$ and Shannon were used as alpha diversity metrics to measure the composition of bacterial communities (Figure 7). The plot was generated by taking the total number of OTUs in a sample by using shiny-phyloseq. Figure 7a represents the alpha diversity abundance value of all 80 biofilm samples. The horizontal $X$-axis is represented by the sample indicated by faint gray lines. Every sample has a separate discrete position on the graph corresponding to the number of OTUs present in that sample. The $Y$-axis represents the number of OTUs, ranging from 200 to 800 values. The Chao $^{1}$ richness estimate had no statistically significant difference between samples. A total of 42,560 OTUs were found across all biofilm samples. The species evenness was estimated using Shannon diversity index, with the horizontal X-axis representing the number of samples. A Shannon index (Figure $7 b$ ) ranging from 5.18 to 6.48 was observed overall, and clustering was detected, indicating an evenness of the bacterial community in all samples.

To determine the difference of dissimilarity in microbial complexes at the species level, the weighted UniFrac distance and Jaccard Index were estimated using QIIME. The principal coordinate analysis estimated the distance between samples and presented them into three visual axes based on the variability between samples. The PCO graphs for each sample are represented by a dot based on their UniFrac distance in the 3D graph and the differential coloring are given for sex (blue and red) and age (green and red) characteristics. Clustering was observed in both age and sex due to the samples containing bacterial community of similar composition. A total variance of $39.19 \%$ ( $r s=0.36, P>0.05)$ was observed for sex (weighted UniFrac) with the first, second, and third axis variability explaining $9.53 \%, 15.05 \%$, and $14.61 \%$ respectively (Figure $8 \mathrm{a}$ ). Regarding age (weighted UniFrac) $15.77 \%$ ( $r s=0.28, \mathrm{P}>0.05$ ) variation was observed with the first, second, and third axis variability explaining $3.48 \%, 7.53 \%$, and $4.76 \%$, respectively (Figure $8 d$ ). The percentage measurement of dissimilarity between the age and 
Table 1- The relative abundance of bacterial species from level 7 categorized into subgingival microbial complexes

\begin{tabular}{|c|c|c|c|c|c|c|}
\hline No & Bacterial Complex & Bacterial Species & $\begin{array}{c}\text { Relative } \\
\text { Abundance in \% } \\
\end{array}$ & $\begin{array}{c}\text { Total abundance in } \\
\text { Percentages }\end{array}$ & T statistics & $P$ value \\
\hline \multirow{3}{*}{1} & \multirow{3}{*}{ Red Complex } & T. Denticola $†$ & 2,95 & \multirow{3}{*}{$5,30 \%$} & \multirow{3}{*}{12,06} & \multirow{3}{*}{0,015} \\
\hline & & P. Gingivalis & 1,64 & & & \\
\hline & & T. Forsythia & 0,71 & & & \\
\hline \multirow{4}{*}{2} & \multirow{4}{*}{ Orange Complex } & Campylobacter Rectus & 1,52 & \multirow{4}{*}{$18,99 \%$} & \multirow{4}{*}{20,37} & \multirow{4}{*}{0,002} \\
\hline & & Fusobacterium Nucleatum $†$ & 11,61 & & & \\
\hline & & P.micros & 0,82 & & & \\
\hline & & P.intermedia & 5,04 & & & \\
\hline \multirow{2}{*}{3} & \multirow{2}{*}{ Purple Complex } & Actinomyces spp. & 3,1 & \multirow{2}{*}{$7,90 \%$} & \multirow{2}{*}{24,35} & \multirow{2}{*}{0,002} \\
\hline & & Veillonella spp. & 4,8 & & & \\
\hline \multirow{4}{*}{4} & \multirow{4}{*}{ Yellow Complex } & S. Gordonii & 1,93 & \multirow{4}{*}{$7,49 \%$} & \multirow{4}{*}{14,67} & \multirow{4}{*}{0,001} \\
\hline & & S. Intermedius & 1,81 & & & \\
\hline & & S. Mitis & 2,03 & & & \\
\hline & & S. Sanguis & 1,72 & & & \\
\hline \multirow{2}{*}{5} & \multirow{2}{*}{ Green Complex } & Capnocytophaga spp. & 1,27 & \multirow{2}{*}{$1,33 \%$} & \multirow{2}{*}{5,57} & \multirow{2}{*}{0,004} \\
\hline & & E.corrodens & 0,06 & & & \\
\hline \multirow{5}{*}{6} & \multirow{5}{*}{ Health-related Species } & Neisseria spp. & 5,53 & \multirow{5}{*}{$16,75 \%$} & \multirow{5}{*}{32,15} & \\
\hline & & Gemella spp. & 3,31 & & & \\
\hline & & Rothia spp. & 3,14 & & & 0,002 \\
\hline & & Kingella spp. & 2,11 & & & \\
\hline & & Corynebacterium spp. & 2,66 & & & \\
\hline 7 & Novel periodontal pathogens & Fretibacterium spp.t & 11,81 & $1734 \%$ & 1406 & 0001 \\
\hline & 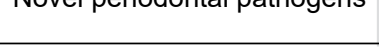 & Saccharibacteria spp. & 5,53 & $11,34 \%$ & 14,00 & 0,001 \\
\hline 8 & Health/Dicease_related & Leptotrichia spp.t & 8,53 & $1561 \%$ & 3274 & 0007 \\
\hline 0 & 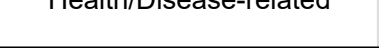 & Selenomonas spp. & 7,08 & 10,0170 & 02,14 & , \\
\hline & & Porphyromonas spp. & 2,65 & & & \\
\hline & & Prevotella spp. & 2,25 & & & \\
\hline & & Treponema spp. & 2,85 & & & \\
\hline & & Odoribacter spp. & 0,25 & & & \\
\hline 9 & ‡Other Species & Johnsonella spp. & 0,07 & $9,29 \%$ & 16,35 & 0,023 \\
\hline & & Oribacterium spp. & 0,02 & & & \\
\hline & & Desulfobulbus & 0,23 & & & \\
\hline & & A. Actinomycetemcomitans & 0,91 & & & \\
\hline & & Wolinella spp. & 0,06 & & & \\
\hline
\end{tabular}

The total abundance in each group was calculated by taking the sum of relative abundance of each bacterial species.

$\dagger$ represents the highest bacterial species in each group.

$\ddagger$ species that has been excluded in the remaining eight groups and their corresponding prevalence indicated greater than $0.1 \%$.

sex for all samples were also measured using Jaccard Index. The percentage of variation was calculated to be $10.69 \%$ and the Jaccard distance was obtained as 0.89. Therefore, a Jaccard index of 0.89 stated an $89 \%$ similarity for two categories of age and sex in Figures $8 \mathrm{~b}$ and $\mathrm{c}$. The results indicated no statistically significant variation between age and sex across all the 80 biofilm samples.

\section{Discussion}

An imbalance in the dynamic relationships among biofilm, host, and microenvironment results in periodontal diseases. ${ }^{13}$ According to the current classification, Stage I periodontitis indicates borderland between gingivitis and periodontitis, Stage II represents established periodontitis with absence of tooth loss and no significant damage to attachment apparatus. ${ }^{15}$ As the underlying cause of 
periodontitis is predominantly microbial, ${ }^{17}$ enumerating the subgingival microbial profile of established periodontitis as in Stage II generalized periodontitis would aid us in deeper understanding of initiation and progression of periodontitis. Stage III and Stage IV periodontitis results in tooth loss and significant damage to the attachment apparatus, ${ }^{15}$ hence the relative abundance of all bacterial healthy/disease

Relative abundance of bacterial complexes in subgingival plaque of

Stage II generalized periodontitis

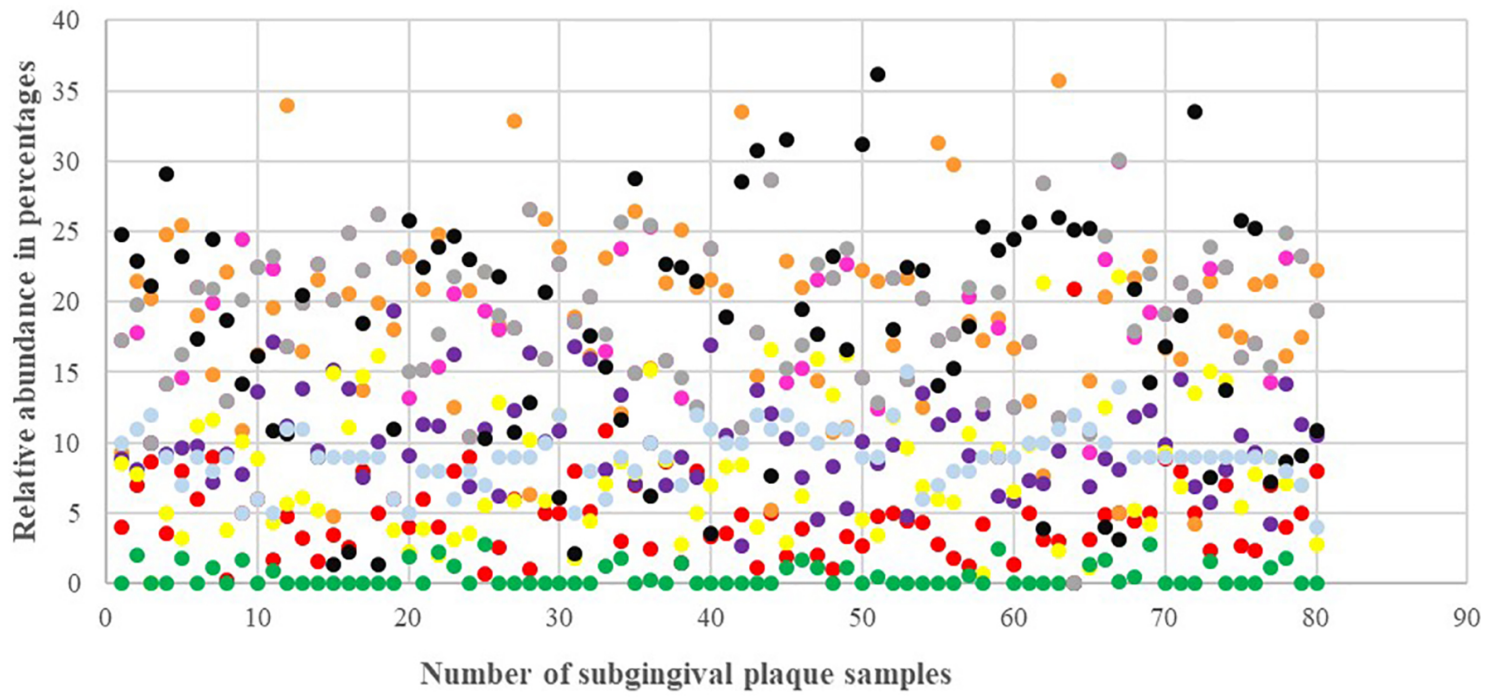

- Red Complex
- Purple Complex
- Green Complex
- Leptotrichia spp. and Selenomonas spp.
- Other species

- Orange Complex

Yellow Complex

- Periodontal health Species

- Novel Periodontal pathogens

Figure 6- The relative abundance of Socransky's bacterial complex and novel bacterial species. The X-axis represents the number of plaque samples, and Y-axis depicts the corresponding percentages

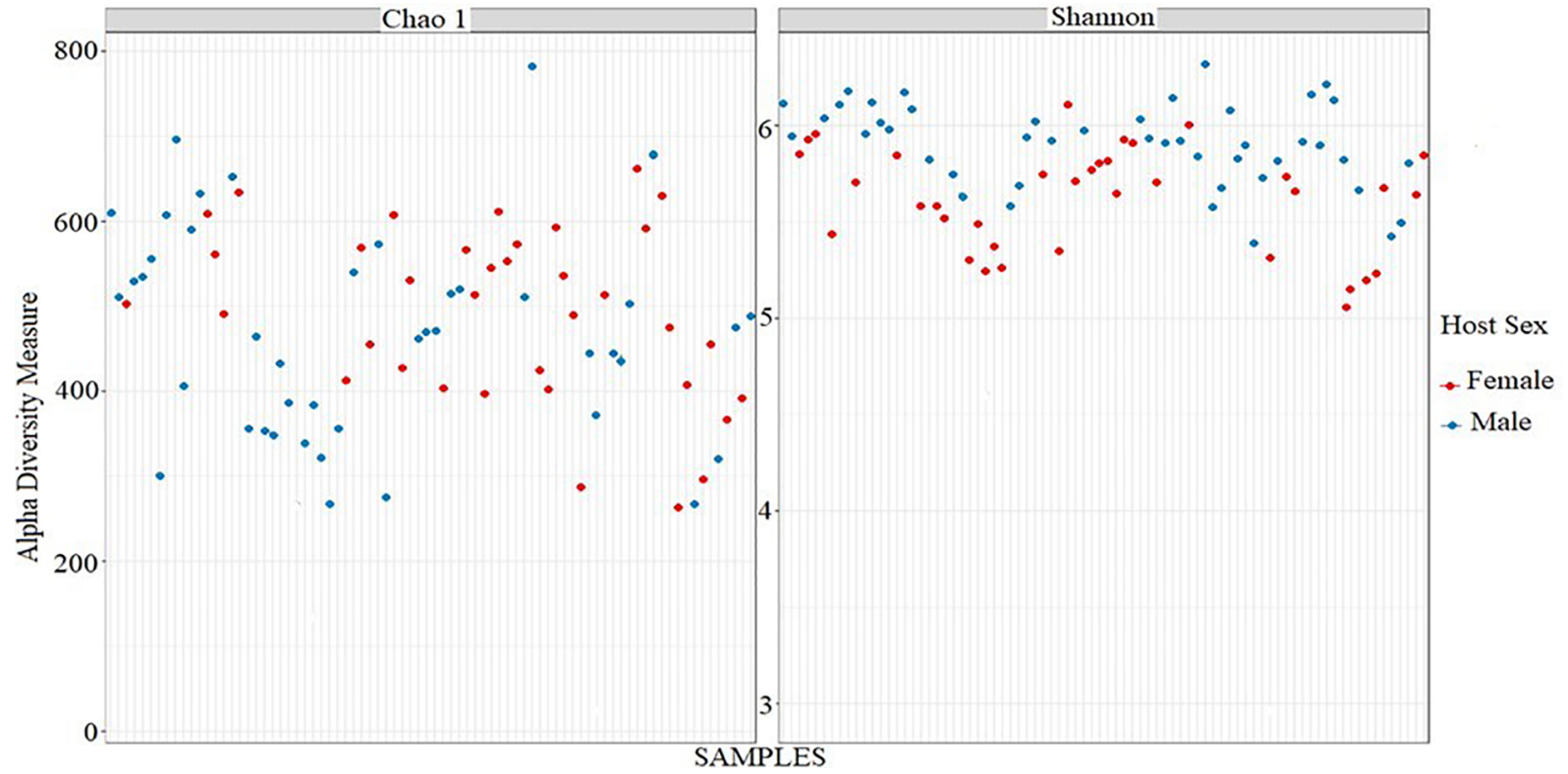

Figure 7- Alpha diversity metrics (a) Chao1 and (b) Shannon Index 

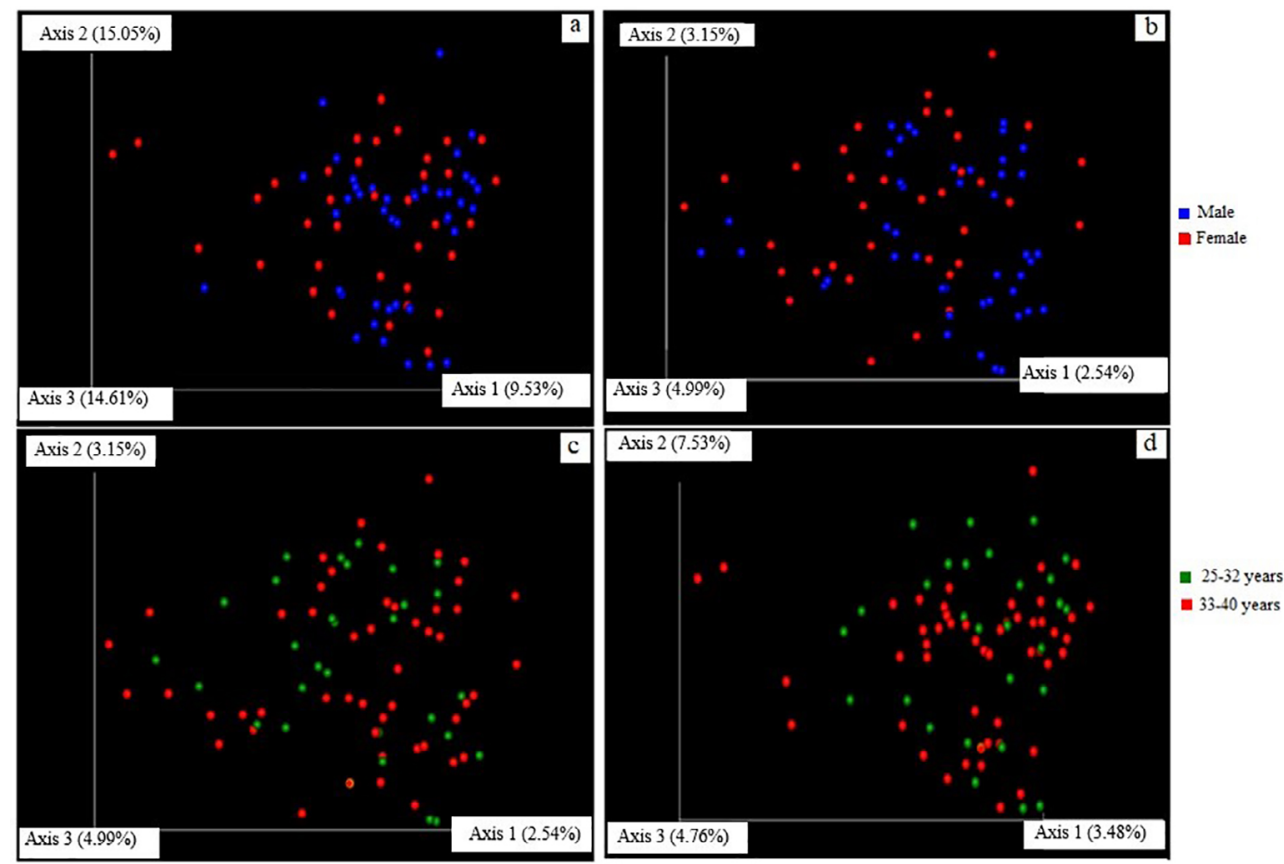

Figure 8- Principal coordinates analysis (PCoA) based on weighted UniFrac distances (a \& d) and Jaccard index (b \& c). PCoA score plot represents the phylogenetic relationship between biofilm samples diagnosed with Stage II generalized periodontitis according to patients' sex (a \& b) and age (c \& d). Axis 1, 2, and 3 are principle coordinates- PC1, PC2, and PC3, respectively

complexes to novel bacterial species may not be accomplished. Since the grade modifiers for Grade A periodontitis are non-smokers and normoglycemic individuals, ${ }^{15}$ patients with a history of smoking and any systemic illness, were excluded from this study. Participants younger than 40 years were included in the study, as advanced periodontal destruction and bone loss are rarely seen in such individuals. ${ }^{18}$

The most predominant phyla observed in the study relating to Stage II generalized periodontitis were Bacteroidetes and Firmicutes. These outcomes were consistent with the previous investigation conducted by Chen, et al. ${ }^{16}$ (2018) on microbial compositional analysis of periodontal diseases. Prevotella and Porphyromonas genera detected in our study represents Bacteroidetes phyla which are Gram-negative facultative anaerobes. They are involved in the formation and progression of several forms of periodontal disease and their high number indicates active periodontal disease. Streptococcus and Gemella genera identified in this study were composed of Gram-positive facultative anaerobes and are classified as firmicutes phyla primarily responsible for periodontal health. Following the above stated phyla, Spirochetes and Fusobacteria indicated a relatively similar frequency in the entire biofilm samples. The most recognized species in Fusobacteria phylum concerning periodontitis is $F$. nucleatum; being prominent quantitatively and the first Gram-negative species to become established in plaque biofilms. ${ }^{19} \mathrm{~A}$ higher presentation of $F$. nucleatum was observed in this study regarding the orange complex. Similarly, the spirochetal accumulation in subgingival plaque is a function of the clinical severity in periodontal disease. ${ }^{20} T$. denticola which has been established by several studies as disease-related complex represents Spirochetes phylum. In our study $T$. denticola outweighed the other bacterial species ( $P$. gingivalis and Tannerella forsythia) in the red complex, indicating a direct co-relation to significantly higher Spirochetes phyla. This observation is in agreement with other studies relating to microbial profiles of periodontal disease. ${ }^{21,22}$

The accompanying phyla identified in Stage II periodontitis along with the above-stated ones, were Proteobacteria, Actinobacteria, Patescibacteria, Epsilonbacteraeota, Synergistetes, and Choloroflexi. In this study, we identified proteobacteria phylum, and their presence was related to periodontal health. Neisseria spp. and Kingella spp. relating to periodontal health were classified under Proteobacteria phylum. Concerning Actinobacteria phyla, Actinomyces spp., Bifidobacterium spp., and Corynebacterium spp., observed in our study were also related to periodontal health. Actinomyces classified under purple complex are related to periodontal health, ${ }^{23}$ Bifidobacterium are involved in preventing periodontal disease ${ }^{24}$ and Corynebacterium are included as health-related 
species. ${ }^{13}$ The prevalence of Proteobacteria and Actinobacteria phyla was comparable to the studies conducted by Shi, et al. ${ }^{25}$ (2018) and Chen, et al.. ${ }^{16}$ (2018), on subgingival plaque microbial profiling. The statistically significant presence of these periodontal health-related bacterial species that prefer an anaerobic environment indicates its presence in the subgingival plaque of Stage II periodontitis.

Patescibacteria - which is present in our study - was proposed by Rinke, et al. ${ }^{26}$ (2013) as a superphylum composed of Gracilibacteria, Microgenomates, and Parcubacteria. In our study we detected a positive co-relation between Gracilibacteria class and Stage II periodontitis. Gracilibacteria was first described in the human oral microbiome in 2014 with a $16 \mathrm{~S}$ rRNA screening, but its relation to periodontitis is still unknown. ${ }^{27}$ Epsilonbacteraeota is a relatively new phyla ${ }^{28}$ - which was previously described as a class of Proteobacteria phylum. Epsilonbacteraeota phyla identified in our study consisted of Wolinella spp. and Campylobacter spp. in the subgingival plaque. Certain Wolinella spp. like Wolinella rectus are associated with adult periodontitis but their role in the disease remains undefined. ${ }^{29} \mathrm{C}$. rectus - which belongs to orange complex - has been detected in our study and the high prevalence of Campylobacter class in fraction per sample was also confirmed. The presence of Synergistetes phyla identified in our research - presenting a stronger association with periodontitis - has been established in investigations conducted by Kirst, et al. ${ }^{9}$ (2015) and Marchesan, et al. ${ }^{30}$ (2015). They have proteolytic properties and are strictly anaerobic bacteria - representing the profiles of periodontal pathogens. Synergistetes species have been associated with gingival bleeding and they prefer a habitat with inflammation and bleeding. ${ }^{31}$ A significant observation in our study was the frequency of Chloroflexi less than $1 \%$ in all the 80 subgingival biofilm samples. These findings contrast with observations in a study conducted by Kirst, et al. ${ }^{9}$ (2015), where these authors detected a relatively high prevalence compared with our study. The suggested cause might be due to the selection of cases involved in the study. This study had taken clinical attachment loss (CAL) of 3-4 mm, whereas their study involved CAL of $\geq 5 \mathrm{~mm}$. The presence of all these anaerobic genera that are mentioned from the phyla Firmicutes, Bacteroidetes, Proteobacteria, Spirochaetes, and Synergistetes constitute a dysbiotic microbial community and these findings were consistent with the current literature. ${ }^{8,12,13}$

A species-level of identification relating to their representative bacterial complex were described in order to recognize their prevalence in Stage II generalized periodontitis. The orange complex, healthrelated species, unclassified health/disease periodontal species, and novel periodontal pathogens represented a higher count, which was statistically significant. This was followed by purple, yellow, red, and green complexes. The health-related species (Neisseria spp., Rothia spp., Kingella spp., and Corynebacterium spp.) and novel species (Fretibacterium spp. and Saccharibacteria spp.) has outnumbered the red complex. A similar observation of the red complex consisting of a relatively small fraction of the total bacterial species was established in a study conducted by Kumar, et al. ${ }^{32}$ (2005). A quantitative 16 S clonal analysis of periodontal pathogens and beneficial species in their study has detected Campylobacter, Abiotrophia, Gemella, Capnocytophaga, and Neisseria genera outnumbering periodontopathogens. Likewise, the presence of a high proportion of putative and novel pathogens in periodontitis has been established by Colombo, et al. ${ }^{13}$ (2019). All these findings propose that the bacterial species belonging to Fretibacterium, Saccharibacteria and other health-related species that are not included in bacterial complexes - play a pivotal role in the dysbiosis of periodontal disease. Though the presence of red complex bacteria is seen in diminished numbers compared to periodontal health contributing species, the presence of clinical attachment loss of $4 \mathrm{~mm}$ could state a foundation for dysbiosis activity in Stage II periodontitis.

The occurrence of all microbial complexes in periodontitis observed in this study constitute an established dysbiosis. ${ }^{13}$ The subgingival plaque composing the microbial complex formed, resembles the sequence of plaque development. The primary colonizers are bacterial species representing the yellow complex. The yellow complex - predominantly composed of Streptococcus species - recognizes the complementary salivary receptors in the acquired pellicle (present on tooth) and provides receptors for secondary colonizers. The secondary colonizers include members of the purple and green complex Actinomyces naes/undii, Capnocytophaga ochracea, Eikenella corrodens, and Veillonella atypica. The primary and secondary colonizers - including 
yellow, purple and green complexes - are the early colonizers. ${ }^{13}$ Succeeding the growth of early colonizers, F. nucleatum colonizes - acting as central species in physical interactions between Gram-positive and Gram-negative species. They act as intermediate colonizers, providing conditions necessary for the emergence of oxygen-intolerant anaerobes. ${ }^{19}$ The red complex considered as the climax community occurring at the final stage uses the anaerobic environment. Thus, during the plaque development, a host-compatible symbiotic relationship gets involved into incipient dysbiotic microflora (gingivitis) leading to a self-resolving inflammatory response. This would further evolve into frank dysbiosis (periodontitis) microflora with ineffective inflammatory responses leading to periodontal tissue destruction. Hence, the presence of bacterial species representing early, intermediate, and climax communities detected in our study, states an established dysbiosis in Stage II periodontitis.

The two novel bacterial species detected in our study were the Fretibacterium spp. and Saccharibacteria spp. The Fretibacterium spp. showed significantly increased levels in the periodontitis cases and were positively correlated with $\geq 4 \mathrm{~mm}$ periodontal pocket depth (PPD) and bleeding on probing (BOP). ${ }^{33}$ As these bacterial species could not be cultivated, their characteristics and virulent aspects relating to the disease progression is unknown. The presence of Fretibacterium was also confirmed in the subgingival plaque of early stages of chronic periodontitis patients. ${ }^{34}$ Saccharibacteria has dynamic interaction with its host bacteria and exhibits virulent killing of host bacterium. These bacteria can inhibit the host's growth dynamics and affect the oral microbial ecology. ${ }^{35}$ Saccharibacteria previously known as TM7 detected in our study were also described by Kirst, et al. ${ }^{9}$ (2015) in periodontitis cases. The role of novel periodontal pathogens has been reported. ${ }^{32}$ These pathogens have shown a strong association with periodontal disease compared to less numerous periodontal pathogens previously implicated.

In addition to the novel species, other relevant species on our study were Neissera spp. Gemella spp., Rothia spp. Kingella spp., and Corynebacterium spp. These bacterial species were clumped together as health-related species based on the review conducted by Colombo, et al. ${ }^{13}$ (2019). Neissera spp. and Gemella spp., are the microbial profile of periodontal health and they were confirmed as beneficial species with quantitative $16 \mathrm{~S}$ Clonal Analysis. ${ }^{32}$ The role of Kingella, Neissera, and Gemella genera in periodontal homeostasis is not yet adequately studied. The presence of these species in higher values compared to the red complex could be clinically manifested as the absence of tooth loss and severe clinical attachment loss. The additional five group of genera identified in this study included Odoribacter, Johnsonella, Oribacterium, Desulfobulbus, and Scardovia. Desulfobulbus and Johnsonella genus were associated with subgingival plaque of periodontitis. ${ }^{16}$ The relative abundance of these genera represented a lower count, and their precise role in periodontitis are unidentified. Nevertheless, their detection has been associated with periodontitis in subgingival plaque. Odoribacter genus, within the family 'Porphyromonadaceae' are anaerobic and Gram-negative - which induced alveolar bone loss in a experiment of periodontal disease with rats. ${ }^{36}$ Oribacterium spp., are strictly anaerobic and Gram-positive species and it has been isolated from human subgingival dental plaque. ${ }^{37}$

The species belonging to Leptotrichia and Selenomonas genera - representing a higher relative abundance -were neither classified as health species nor pathogens as their role in either situation remains uncertain. Leptotrichia spp. are associated with both disease and normal health, representing distinct pathogenic potentials of bacteria in the same genera. ${ }^{16}$ Selenomonas species have been strongly associated with periodontitis, and they play a role in the onset and progression of the disease. Higher levels of Selenomonas Sputigena were detected in the subgingival biofilm of deep periodontal pockets $\leq$ $6 \mathrm{~mm}$, that represents Stage II \& III periodontitis. ${ }^{38}$ However, their exact role in inducing periodontal destruction remains undetected, a higher prevalence of Selenomonas species identified in this study directly associates these species' presence in the periodontal pocket of Stage II generalized periodontitis.

Apart from the role of periodontal microbes, it is essential to consider the host-microbial interaction for the development and manifestation of periodontitis. According to the ecological plaque hypothesis, the host's inflammatory responses and not the specific bacteria and putative virulence factor determines periodontitis development and progression. ${ }^{39}$ Moreover, the nutrient profile of the gingival crevice due to Gingival Cervicular Fluid (GCF) secretion produces a shift in the balance of resident microflora, leading to increased plaque 
biomass and high levels of obligate anaerobic Gramnegative bacteria. ${ }^{40}$ Thus the commensal flora would change to an opportunistic pathogenic flora by complex changes in the local environment, which are primarily driven by the host and not by the bacteria. However, it is noteworthy that the role of subgingival microflora in the etiology and pathogenesis of periodontitis is not diminished.

The beta-diversity measurement based on Unweighted UniFrac distance indicated that the distance between two different age groups was minimal. Although a total variance of $39.19 \%$ was observed for sex, the findings were not statistically significant. While bacterial species variation occurs in males and females, a non-significant difference in this study could have resulted from the exclusion of subjects who presented systemic conditions. Systemic conditions like pregnancy would induce hormonal changes and variation in bacterial species compared to male subjects. A Jaccard Index of $89 \%$, similar in both age and sex, suggests that the microbial profile did not vary between biofilm samples and are representative of Stage II generalized periodontitis.

The 16s rRNA based microbial profiling via NGS in periodontal conditions is the first of its kind to be studied in the United Arab Emirates. Exact quantification of bacterial species less than $0.01 \%$ has not been described in our study, which could be a limitation. Also,16-23S rRNA providing high-resolution analyses for bacterial species identification has not been employed in our study. However, 16S rRNA-based bacterial profiling via next-generation sequencing of supragingival and subgingival plaque has been employed in previous studies to determine the composition and diversity of bacterial communities. ${ }^{16,25}$ Based on our study, it is possible to suggest that there is an association between Stage II generalized periodontitis and novel periodontal pathogens. Future studies based on proteomic and metabolomic analyses would provide information on the interbacterial relationship of these novel periodontal pathogens and improve our understanding on microbial function in periodontal pathogenesis.

\section{Conclusion}

The 16S rRNA via NGS approach in patients diagnosed with Stage II generalized periodontitis indicated that the bacterial species belonging to the orange-complex and novel periodontal pathogens are the most abundant.

\section{Acknowledgments}

We would like to thank Agiomix Lab, Dubai science park, UAE, for their support in conducting the metagenomics analysis and their inputs

\section{Funding and sponsorship}

This study has been supported by the deanship of graduate studies and research, Ajman University of Science and Technology, Ajman, UAE. (Reference No-2017-A-DN-04)

\section{Conflicts of interest}

There are no conflicts of Interest.

\section{Authors' contributions}

Abu Fanas, Salim: Conceptualization (Equal); Formal analysis (Equal); Funding acquisition (Equal); Methodology (Equal); Resources (Equal); Validation (Equal); Writing-original draft (Equal). Brigi, Carel: Formal analysis (Equal); Funding acquisition (Equal); Investigation (Equal); Methodology (Equal); Project administration (Equal); Validation (Equal); Writingoriginal draft (Equal); Writing-review \& editing (Equal). Varma, Sudhir Rama: Conceptualization (Equal); Formal analysis (Equal); Funding acquisition (Equal); Investigation (Equal); Validation (Equal); Writingoriginal draft (Equal); Writing-review \& editing (Equal). Desai, Vijay: Conceptualization (Equal); Formal analysis (Equal); Investigation (Equal); Methodology (Equal); Validation (Equal); Writing-original draft (Equal). Senok, Abiola: Investigation (Equal); Methodology (Equal); Supervision (Equal); Validation (Equal); Writing-original draft (Equal); Writing-review \& editing (Equal). D'Souza, Jovita: Conceptualization (Equal); Methodology (Equal); Project administration (Equal); Supervision (Equal); Validation (Equal); Writing-original draft (Equal).

\section{References}

1- Hall MW, Singh N, Ng KF, Lam DK, Goldberg MB, Tenenbaum HC, et al. Inter-personal diversity and temporal dynamics of dental, tongue, and salivary microbiota in the healthy oral cavity. NPJ Biofilms Microbiomes. 2017;26(3):2. doi: 10.1038/s41522-016-0011-0 
2- Payne MA, Hashim A, Alsam A, Joseph S, Aduse-Opoku J, Wade WG, et al. Horizontal and vertical transfer of oral microbial dysbiosis and periodontal disease. J Dent Res. 2019;98(13):1503-10. doi: $10.1177 / 0022034519877150$

3- Deng ZL, Szafrański SP, Jarek M, Bhuju S, Wagner-Döbler I. Dysbiosis in chronic periodontitis: key microbial players and interactions with the human host. Sci Rep. 2017;7(1):3703. doi: 10.1038/s41598017-03804-8

4- Hajishengallis G. Dysbiosis and inflammation in periodontitis: synergism and implications for treatment. J Oral Microbiol. 2017;9 Suppl 1):1325198. doi: 10.1080/20002297.2017.1325198

5- Socransky SS, Haffajee AD, Cugini MA, Smith C, Kent RL Jr. Microbial complexes in subgingival plaque. J Clin Periodontol. 1998;25(2):13444. doi: 10.1111/j.1600-051x.1998.tb02419.x

6- Boutaga K, van Winkelhoff AJ, Vandenbroucke-Grauls CM, Savelkoul $\mathrm{PH}$. The additional value of real-time PCR in the quantitative detection of periodontal pathogens. J Clin Periodontol. 2006;33(6):427-33. doi: 10.1111/j.1600-051X.2006.00925.x

7- Hajishengallis G, Lamont RJ. Beyond the red complex and into more complexity: the polymicrobial synergy and dysbiosis (PSD) model of periodontal disease etiology. Mol Oral Microbiol. 2012;27(6):409-19. doi: 10.1111/j.2041-1014.2012.00663.x

8- Hajishengallis G. Periodontitis: from microbial immune subversion to systemic inflammation. Nat Rev Immunol. 2015;15(1):30-44. doi: 10.1038/nri3785

9- Kirst ME, Li EC, Alfant B, Chi YY, Walker C, Magnusson I, et al. Dysbiosis and alterations in predicted functions of the subgingival microbiome in chronic periodontitis. Appl Environ Microbiol. 2015;81(2):783-93. doi: 10.1128/AEM.02712-14

10- Colombo AP, Bennet S, Cotton SL, Goodson JM, Kent R, Haffajee $A D$, et al. Impact of periodontal therapy on the subgingival microbiota of severe periodontitis: comparison between good responders and individuals with refractory periodontitis using the human oral microbe identification microarray. J Periodontol. 2012;83(10):1279-87. doi: 10.1902/jop.2012.110566

11- Jiao $Y$, Hasegawa $M$, Inohara N. The role of oral pathobionts in dysbiosis during periodontitis development. J Dent Res. 2014;93(6):539-46. doi: 10.1177/0022034514528212

12- Pérez-Chaparro PJ, Gonçalves C, Figueiredo LC, Faveri M, Lobão E, Tamashiro N, et al. Newly identified pathogens associated with periodontitis: a systematic review. J Dent Res. 2014;93(9):846-58. doi: $10.1177 / 0022034514542468$

13- Colombo AP, Tanner AC. The role of bacterial biofilms in dental caries and periodontal and peri-implant diseases: a historical perspective. J Dent Res. 2019;98(4):373-85. doi: 10.1177/0022034519830686 14- Wei Y, Shi M, Zhen M, Wang C, Hu W, Nie Y, et al. Comparison of subgingival and buccal mucosa microbiome in chronic and aggressive periodontitis: a pilot study. Front Cell Infect Microbiol. 2019;9:53. doi: 10.3389/fcimb. 2019.00053

15- Tonetti MS, Greenwell H, Kornman KS. Staging and grading of periodontitis: framework and proposal of a new classification and case definition. J Periodontol. 2018;89(1):S159-72. doi: 10.1002/ JPER.18-0006

16- Chen WP, Chang SH, Tang CY, Liou ML, Tsai SJ, Lin YL. Composition analysis and feature selection of the oral microbiota associated with periodontal disease. Biomed Res Int. 2018;2018:3130607. doi: $10.1155 / 2018 / 3130607$

17- Berezow AB, Darveau RP. Microbial shift and periodontitis Periodontol 2000. 2011;55(1):36-47. doi: 10.1111/j.16000757.2010.00350.x

18- AlJehani YA. Risk factors of periodontal disease: review of the literature. Int J Dent. 2014;2014:182513. doi: 10.1155/2014/182513 19- Signat B, Roques C, Poulet P, Duffaut D. Fusobacterium nucleatum in periodontal health and disease. Curr Issues Mol Biol. 2011;13(2):2536.
20- Loesche WJ. The role of spirochetes in periodontal disease. Adv Dent Res. 1988;2(2):275-83. doi: 10.1177/08959374880020021201 21- Reed LA, O'Bier NS, Oliver LD Jr, Hoffman PS, Marconi RT. Antimicrobial activity of amixicile against Treponema denticola and other oral spirochetes associated with periodontal disease. J Periodontol. 2018;89(12):1467-74. doi: 10.1002/JPER.17-0185 22- Tanno-Nakanishi M, Kikuchi Y, Kokubu E, Yamada S, Ishihara $\mathrm{K}$. Treponema denticola transcriptional profiles in serum-restricted conditions. FEMS Microbiol Lett. 2018;365(16):fny171. doi: 10.1093/ femsle/fny 171

23- Popova C, Dosseva-Panova V, Panov V. Microbiology of periodontal diseases: a review. Biotechnol Biotechnol Equip. 2013;27(3): 3754-9. doi: 10.5504/BBEQ.2013.0027

24- Hojo K, Mizoguchi C, Taketomo N, Ohshima T, Gomi K, Arai $\mathrm{T}$, et al. Distribution of salivary Lactobacillus and Bifidobacterium species in periodontal health and disease. Biosci Biotechnol Biochem. 2007;71(1):152-7. doi: 10.1271/bbb.60420

25- Shi M, Wei Y, Hu W, Nie Y, Wu X, Lu R. The subgingival microbiome of periodontal pockets with different probing depths in chronic and aggressive periodontitis: a pilot study. Front Cell Infect Microbiol. 2018;8:124. doi: 10.3389/fcimb.2018.00124

26- Rinke C, Schwientek P, Sczyrba A, Ivanova NN, Anderson IJ, Cheng $\mathrm{JF}$, et al. Insights into the phylogeny and coding potential of microbial dark matter. Nature. 2013;499(7459):431-7. doi: $10.1038 /$ nature 12352

27- Camanocha A, Dewhirst FE. Host-associated bacterial taxa from Chlorobi, Chloroflexi, GN02, Synergistetes, SR1, TM7, and WPS-2 Phyla/candidate divisions. J Oral Microbiol. 2014;6. doi: 10.3402/ jom.v6.25468

28- Waite DW, Vanwonterghem I, Rinke C, Parks DH, Zhang Y, Takai K, et al. Comparative genomic analysis of the class Epsilonproteobacteria and proposed reclassification to Epsilonbacteraeota (phyl. nov.). Front Microbiol. 2017;8:682.

doi: $10.3389 /$ fmicb. 2017.00682

29- Lai CH, Oshima K, Slots J, Listgarten MA. Wolinella recta in adult gingivitis and periodontitis. J Periodontal Res. 1992;27(1):8-14. doi: 10.1111/j.1600-0765.1992.tb02079.x

30- Marchesan JT, Morelli T, Moss K, Barros SP, Ward M, Jenkins W, et al. Association of Synergistetes and Cyclodipeptides with periodontitis. J Dent Res. 2015;94(10):1425-31. doi: 10.1177/0022034515594779 31- Vartoukian SR, Palmer RM, Wade WG. Diversity and morphology of members of the phylum "synergistetes" in periodontal health and disease. Appl Environ Microbiol. 2009;75(11):3777-86. doi: 10.1128/ AEM.02763-08

32- Kumar PS, Griffen AL, Moeschberger ML, Leys EJ. Identification of candidate periodontal pathogens and beneficial species by quantitative 16 S clonal analysis. J Clin Microbiol. 2005;43(8):3944-55. doi: 10.1128/JCM.43.8.3944-3955.2005

33- Khemwong $T$, Kobayashi $H$, Ikeda $Y$, Matsuura T, Sudo T, Kano C, et al. Fretibacterium sp. human oral taxon 360 is a novel biomarker for periodontitis screening in the Japanese population. PLoS One. 2019;14(6):e0218266. doi: 10.1371/journal.pone.0218266 34- Brinig MM, Lepp PW, Ouverney CC, Armitage GC, Relman DA. Prevalence of bacteria of division TM7 in human subgingival plaque and their association with disease. Appl Environ Microbiol. 2003;69(3):1687-94. doi: 10.1128/aem.69.3.1687-1694.2003 35- Bor B, Bedree JK, Shi W, McLean JS, He X. Saccharibacteria (TM7) in the human oral microbiome. J Dent Res. 2019;98(5):500-9. doi: $10.1177 / 0022034519831671$

36- Hardham JM, King KW, Dreier K, Wong J, Strietzel C, Eversole RR, et al. Transfer of Bacteroides splanchnicus to Odoribacter gen. nov. as Odoribacter splanchnicus comb. nov., and description of Odoribacter denticanis sp. nov., isolated from the crevicular spaces of canine periodontitis patients. Int J Syst Evol Microbiol. 2008;58(Pt 1):103-9. doi: $10.1099 /$ ijs.0.63458-0 
37- Sizova MV, Muller PA, Stancyk D, Panikov NS, Mandalakis M, Hazen A, et al. Oribacterium parvum sp. nov. and Oribacterium asaccharolyticum sp. nov., obligately anaerobic bacteria from the human oral cavity, and emended description of the genus Oribacterium. Int J Syst Evol Microbiol. 2014;64(Pt 8):2642-49. doi: 10.1099/ ijs.0.060988-0

38- Gonçalves LF, Fermiano D, Feres M, Figueiredo LC, Teles FR, Mayer MP, et al. Levels of Selenomonas species in generalized aggressive periodontitis. J Periodontal Res. 2012;47(6):711-8. doi: 10.1111/j.1600-0765.2012.01485.x
39- Bartold PM, Van Dyke TE. Periodontitis: a host-mediated disruption of microbial homeostasis. Unlearning learned concepts. Periodontol 2000. 2013;62(1):203-17. doi: 10.1111/j.1600-0757.2012.00450.x 40- Marsh PD. Microbial ecology of dental plaque and its significance in health and disease. Adv Dent Res. 1994;8(2):263-71. doi: $10.1177 / 08959374940080022001$ 